\title{
ASPERGILOSIS PULMONAR EN CHILE ENFOQUE INMUNOLOGICO
}

Clara Retamal, Cristina Díaz, Lucía Salamanca

Unidad de Microbiología y Parasitología, Fac. de Med.

Div. Cs. Méd. Oriente, Univ. de Chile, Santiago.

Luis Ferrada

Unidad de Microbiología, Fac. Med. Div. Cs. Méd. Norte Universidad de Chile, Santiago.

Raúl Alvarez de Oro Instituto Nac. de Enfermedades Respiratorias y Cirugía Torácica (I.N.E.R. y C.T.), Santiago

\section{RESUMEN}

Las micosis pulmonares por hongos oportunistas presentan frecuentemente cuadros clinicos y signos radiológicos de diagnóstico diferencial dificil con otras patologias broncopulmonares crónicas.

En este estudio corroboramos la existencia de la Aspergilosis pulmonar en Chile, efectuando sistemáticamente las pruebas de inmunoprecipitación en agarosa y estudio micológico en pacientes con sospecha clínica de aspergilosis.

De 850 pacientes estudiados se diagnosticó la Aspergilosis broncopulmonar, clinica e inmunológicamente, en 132 de ellos $(15,5 \%)$.

El $82 \%$ de los casos parasitados correspondió a pacientes con secuelas tuberculosas inactivas previas, $y$ el 14\% a formas hipersensitivas, observándose una clara correlación entre las formas clínicas de Aspergilosis y el estudio radiológico, inmunológico y micológico.

El agente causan te más comín correspondió a Aspergillus fumigatus, $y$ en orden decreciente $A$. flaves, A. niger, $A$ nidulans y $A$. terreus.

Este trabajo pone en evidencia la importancia del diagnóstico inmunológico y su excelente especificidad en relación al cultivo micológico de expectoración en pacientes con patologia pulmonar predisponente, y su utilidad en el seguimiento y pronóstico de estas micosis.

\section{INTRODUCCION ,}

La infección respiratoria por hongos del género Aspergillus, constituye con frecuencia un importante problema clínico y epidemiológico en nuestro medio.

Diagnosticada en varias partes del mundo, no parecía constituir un problema serio de patología en Chile. Sin embargo, sabemos que las micosis

\section{SUMMARY}

[Pulmonary Aspergillosis in Chile an immunological scope]

Pulmonary mycoses caused by opportunistic fungi usually present a difficult problems in the differential diagnosis of chronic bronchopulmonary diseases, both clinical and radiological. In this study we verified the incidence of pulmonary aspergillosis in Chile by systematic performing immunoprecipitation test in agarose. In 850 patients studied, bronchopulmonary aspergillosis was diagnosed in 132 by their clinical and immunologic methods.

$82 \%$ of these cases were patients with inactive tuberculosis, $14 \%$ were hipersensitivity forms. There was a clear correlation between the clinical presentation and radiologic, immunologic and mycologic studies.

The most common agent was Aspergillus fumigatus. Less frequently and in decreasing frecquency, $A$. flavus, $A$. niver, $A$. nidulans and $A$. terreus were identified.

This paper emphasizes the importance of immunologic diagnosis and its excellent specificity, as compared to mycologic sputum cultures in patients with predisposing pulmonary diseases and its usefulness in the follow-up and prognosis of these mycoses.

pulmonares presentan sintomatología clínica e imágenes radiológicas, indiferenciables a veces de enfermedades producidas por otros agentes infecciosos o cáncer pulmonar.

Esta forma de micosis profunda, es producida por un grupo de especies del género Aspergillus, hongos oportunistas de amplia distribución en la naturaleza, saprófitos ubicuos, y cuyo conocimiento sobre su patogenicidad adquirida, data desde muchas décadas. 
Investigadores como Pepys, Biguet y otros, (3-5-6) fueron los que dieron gran impulso al inmunodiagnóstico de la Aspergilosis humana, aplicando técnicas especiales para el estudio de esta enfermedad (7). En el año 1972, Yarzábal (13) introduce en nuestro medio las reacciones de inmunoprecipitación en agarosa, técnicas de gran sensibilidad y especificidad tanto para el diagnóstico de parasitosis como de micosis (2-14-15).

Como para todas las enfermedades infecciosas, el diagnóstico de una micosis, depende del aislamiento del hongo y de su identificación en el individuo infectado, pero también de los mediadores de la respuesta inmune específica, tanto humoral, como celular, es decir, anticuerpos y células sensibilizadas.

El primer criterio, que constituye el estudio por cultivo micológico, el único disponible hasta hace pocos años, no siempre proporciona un diagnóstico definitivo, por tratarse de agentes cuyas esporas pueden inhalarse y expectorarse sin causar patología, tal como sucede con todos los hongos ambientales $(10-11)$.

Complementando la clínica y el estudio micológico, con las técnicas indirectas de diagnóstico, como son las reacciones de inmunoprecipitación en gel, que nos permiten visualizar anticuerpos específicos antiaspergilares, ha sido posible demostrar una prevalencia importante de esta micosis oportunista en Chile en estos últimos años $(1-13)$. Por tanto, el objetivo de este trabajo es analizar los hallazgos inmunológicos en pacientes con antecedentes pulmonares crónicos, su relación con las formas clínicas, los cultivos micológicos y su evolución en los pacientes tratados quirúrgicamente.

\section{MATERIAL Y METODO}

Este estudio correspondió a 850 pacientes con patología broncopulmonar crónica, especialmente broncorrea $y / o$ hemoptisis a repetición, que consultaron en su mayoría en el Area Oriente de Santiago, durante los años 1975 a 1980.

En todos ellos se investigó en el suero, la presencia de precipitinas antiaspergilares, empleando 3 técnicas de inmunoprecipitación en Agarosa. Inmunodifusión doble (DDA), como screening, y para complementar el estudio inmunológico la Electrosinéresis (E.S.) e Inmunoelectroforesis (IEF) unidimensional.

Como antígeno empleamos extractos metabólicos y somático de las especies de Aspergillus fumigatus, A. flavus, A. effusus, A. niger, A. nidulans y A. terreus, para cuya preparación seguimos las normas dadas por el Center for Disease Control (CDC) de Atlanta (7-10).

Estos antigenos se usaron liofilizados y a una concentración de $200 \mathrm{mg} / \mathrm{ml}$ con buffer Veronal pH 8,2 y como soporte, agarosa al $1 \%$ en el mismo buffer.
En el $80 \%$ de los pacientes fue posible realizar paralelamente el estudio micológico seriado de la expectoración, $y$ en alrededor de un $10 \%$ de ellos se efectuó cultivo de secreción broncoaspirada.

\section{RESULTADOS}

En 132 de los 850 pacientes estudiados, $(15,5 \%)$, detectamos un mínimo de 2 bandas de precipitación con las 3 técnicas empleadas, frente a los diferentes antigenos aspergilares.

Las edades de los pacientes flucturaron entre los 18 y 70 años, pero la mitad de ellos tenía entre 30 y 50 años; perteneciendo dos tercios al sexo masculino.

Sabemos que la frecuencia más alta de infección Aspergilar se encuentra asociada fundamentalmente a la tuberculosis pulmonar inactiva (4-9). En este estudio el $82 \%$ de los casos correspondió a parasitación secundaria a secuelas cavitarias tuberculosas, observando que el tiempo transcurrido entre la tuberculosis pulmonar como lesión primaria y la colonización por Aspergillus, fluctuó entre 6 meses y 10 años. Sin embargo, la tuberculosis activa no excluye la micosis, ya que en 4 pacientes se observó baciloscopía positiva, cultivo de hongos y serología también positiva. Otras patologías concomitantes encontradas fueron cancer, bronquiectasia, enfermedad obstructiva crónica y otras. (Tabla 1)

Nosotros observamos en el $53 \%$ de los pacientes la forma Intracavitaria o Aspergiloma atipico $y$ en el $32 \%$ Aspergiloma típico. En estos últimos, la imágen radiológica se visualiza como una masa redondeada, dentro de una cavidad, con un halo periférico o "fungus-ball". En ambos grupos identificamos entre 3 y 14 arcos de precipitación, y en el $95 \%$ de los casos específicos a Aspergillus fumigat us observamos la presencia del sistema precipitante C por Inmunoelectroforesis (diagrama I, II, III). El $14 \%$ restante correspondió a cuadros bronquiales obstructuvos crónicos con sintomas y signos de hipersensibilidad a antígenos aspergilares (aspergilina positiva) y, en algunos de ellos, sin hongo demostrable (Tabla 2). En estos casos el número de arcos visualizados por la I.E.F. en el suero, fue claramente menor (1 ó 2), no observándose en ellos anticuerpos del sistema precipitante $\mathrm{C}(10-15)$.

Se sabe que la especie del género Aspergillus que provoca patología pulmonar con mayor frecuencia es el A. fumigatus $(1-7$ - 9 - 11); en nuestro estudio, lo detectamos en el $80,3 \%$ de los pacientes con aspergilosis, seguido por A. flavus $(10,6 \%)$ $y$ en orden decreciente las especies de A. effusus, A. niger, A. nidulans y $A$. terreus.

Llama la atención el número importante de pacientes parasitados por A. flavus, en comparación con la proporción encontrada en otras regiones del mundo, que no sobrepasa el $2 \%(6-12)$ (Tabla 3). 
TABLA I

DISTRIBUCION DE 132 CASOS DE ASPERGILOSIS SEGUN PATOLOGIA PULMONAR PREVIA

\begin{tabular}{lcr}
\hline & $\mathrm{N}^{\circ}$ Casos & $\%$ \\
\hline $\begin{array}{l}\text { Secuelas tuberculosas } \\
\text { inactivas }\end{array}$ & 108 & 81,8 \\
$\begin{array}{l}\text { Tuberculosis activa } \\
\begin{array}{l}\text { Enfermedad Bronquial } \\
\text { Obstructiva Crónica }\end{array}\end{array}$ & 4 & 3,0 \\
$\begin{array}{l}\text { Otras Patologías } \\
\text { (Bronquiectasia - quiste } \\
\text { broncogénico cáncer, etc.) }\end{array}$ & 6 & 4,5 \\
\hline
\end{tabular}

TABLA 2

FORMAS CLINICAS EN LOS 132 PACIENTES CON SEROLOGIA POSITIVA ANTIASPERGILAR

\begin{tabular}{lcc}
\hline & N $^{\circ}$ Casos & $\%$ \\
\hline $\begin{array}{l}\text { Aspengilosis intracavitaria } \\
\text { atípica }\end{array}$ & 70 & 53,0 \\
$\begin{array}{l}\text { Aspergiloma típico } \\
\text { (“fungus - ball") }\end{array}$ & 42 & 31,8 \\
Formas hipersensitivas & 19 & 14,4 \\
Septicemia aspergilar & 1 & 0,8 \\
\hline
\end{tabular}

TABLA 3

PRECIPITINAS DETECTADAS EN LOS 132 PACIENTES FRENTE A ANTIGENOS ASPERGILARES

\begin{tabular}{lrr}
\hline Antígenos Aspergilares & No Casos & $\%$ \\
\hline Aspergilus fumigatus & 106 & 80,3 \\
Aspergillus flavus & 14 & 10,6 \\
Aspergillas effusus & 5 & 3,8 \\
Aspergillus niger & 4 & 3,0 \\
Aspergillus nidulans & 2 & 1,5 \\
Aspergillus terreus & 1 & 0,8 \\
\hline
\end{tabular}

En las muestras analizadas, el cultivo micológico reveló un $69 \%$ de positividad en relación a la confirmación clínica e inmunológica.

De estos pacientes estudiados, 52 fueron resecados quirúrgicamente (39\%). Fue posible efectuar seguimiento clínico y serológico en 15 de ellos (Gráfico 1), observándose disminución paulatina del número de arcos de precipitación hasta llegar a una negativización serológica, en un plazo que fluctuó entre 6 meses y 2 años.

\section{CONCLUSIONES}

La alta prevalencia de esta micosis profunda en el grupo de pacientes estudiados en 6 años de observación nos permite afirmar que es una patología frecuente y que debe tenerse en consideración en aquellos enfermos broncopulmonares crónicos, $y$ en los que han sido sometidos a terapia que deprima la respuesta inmune humoral o celular.

El empleo de antígeno estandarizado de buena calidad y preparados en nuestro medio, con cepas patógenas chilenas, ha permitido identificar en el suero de la mayoría de estos pacientes, sistemas antígeno anticuerpo especícicos de especie que dan un diagnóstico etiológico seguro de la Aspergilosis broncopulmonar.

El diagnóstico de esta micosis en un ex tuberculoso mediante estudios aislados o seroepidemiológicos, puede detectar precozmente esta parasitación, y permitir al clínico plantear un tratamiento oportuno $(9-11)$.

La determinación secuencial del número de Anticuerpos precipitantes, tiene valor pronóstico, ya que las bandas disminuyen progresivamente hasta desaparecer después de una intervención quirúrgica.

Finalmente, el empleo generalizado de las técnicas de inmunodifusión a nivel de los Laboratorios Clínicos Asistenciales del país, y si es posible su complementación con otras técnicas, puede contribuir en gran manera al mejor conocimiento de esta micosis como un problema más de Salud Pública (15). 


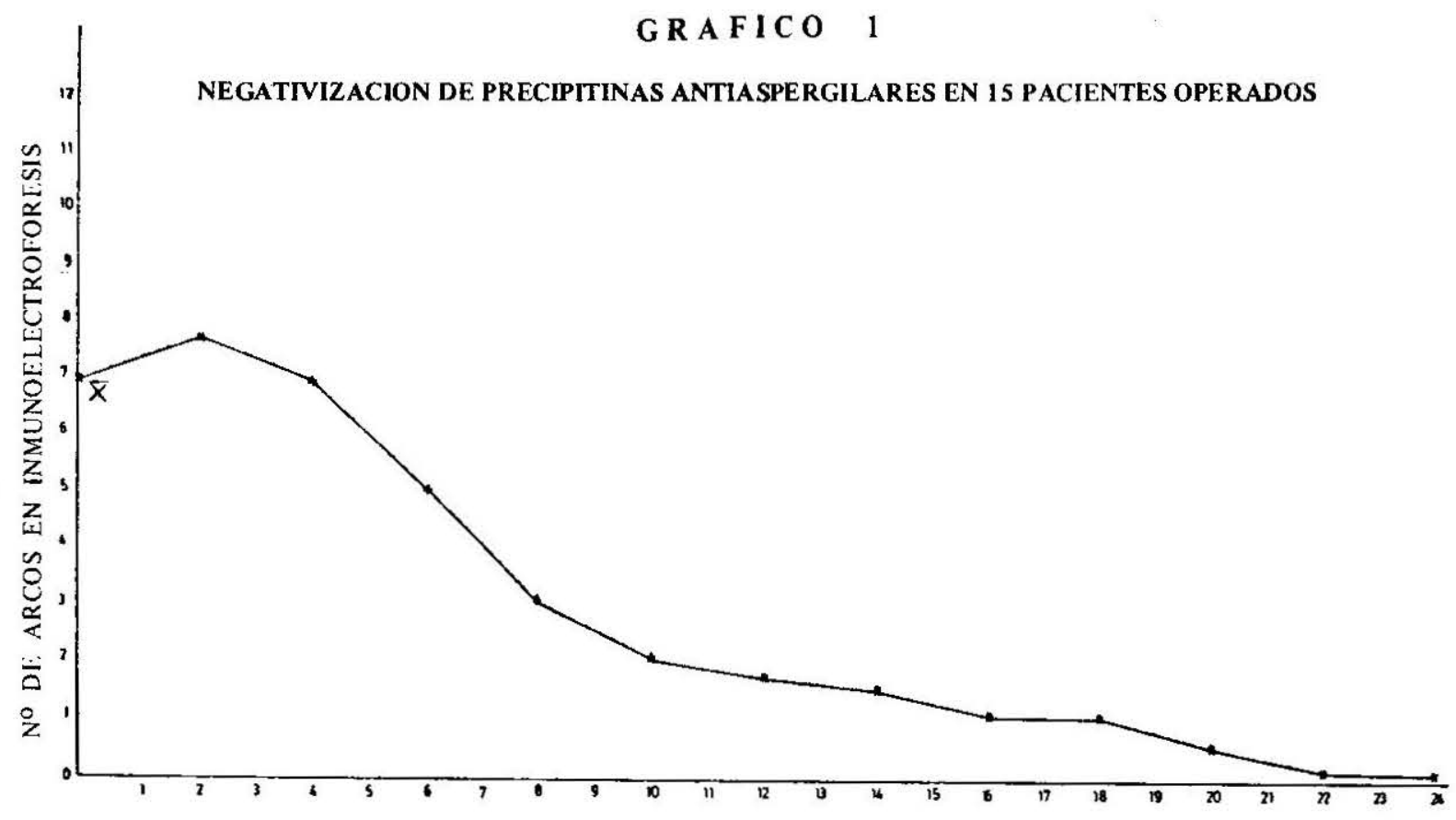

M L S F S

\section{B I B L I O G R A F I A}

1.- Alvarez de Oro, R., Aguilar, H., Contreras, B., Retamal C. y Sepúlveda, R. (1976). Cirugía del Aspergiloma Pulmonar. Rev. Chilena de Cirugia. 28: 19-21.

2.- Biguet, S., Fruit, S., Andrieu, S., Travanki, P. (1969). Fstructura antigénica $y$ sistematica de especies del género Aspergillius. Bull. Soc. Mycol. France, 85: $273-282$. -

3.- Drohuet, E., Segretain, G. et Biguet, R. (1965). Etude des precipitines seriques an milieu gélosé pour le diagnostique des Aspergillosis broncho-pulmonaires. Ann. Inst. Pasteur (Paris) 105: 596-602.

4... Davies, D. (1970). Aspergiloma is residual pulmonary lesion report on a study in Great Britain. Bull. U. Int. Tuberc. $43: 115-120$.
5.- Kaufman, L. (1973). Value of inmunodiffusion tests in the diagnosis of systemic mycotic diseases. S. Clin. Sci. 3: 141-148.

6. - Longbotton, J. and Pepys, J. (1964). Diagnostic precipitin test in Aspergillus pulmonary micetoma Lancet $1: 588-589$.

7. Longbotton, J. and Pepys, J. (1964). Pulmonary Aspergillosis diagnostic and inmunological significances of antigens and C. Substance in Aspergillus fumigatus. J. Pathol. Bacteriol. $88: 141-149$.

8. Mc Carthy, D.S., Pepys, J. (1971). Allergy broncho pulmonary Aspergillosis. Clinical Immunology 1 . Clinical features. Clin. Allerg. 1: 261-270. 
9.- Research Committee of the B.T.A. (1968). Aspergillus in persistent lung cavities after tuberculosis. Tubercle (London) $49: 10$.

10.- Sang, S., Sotirosd, Ch. (1978). Characterization of antigens from Aspergillus fumigatus. Am. Rev. Resp. Dis. 118 : $547-551$.

11.- Voison, C., Biguet, J. (1970). L'aspergillose dans les lesions pulmonaires residuelles; problems diagnostiques, pronostiques et therapeutiques. Bull. U. Int. Tuberc. $43: 119-125$.

12.- Villar, G., Carper Pimentel, S., Avila, R. (1967) Some Aspecto of Pulmonary Aspergilloma in Portugal. Dis. Chest. $51:$ 402-405.
13. - Yarzabal, L., Sepúlveda, R., Retamal, C., Kinzel, R., Castro, M., Salamanca, L. (1974). Aspergillosis respiratoria humana. Rev. Med. Chile. 102: 772-776.

14. - Yarzabal, L., Da Luz, S., Josef, M., y cols. (1973). Pruebas de Inmunoprecipitación en el diagnóstico de la Aspergillosis. Rev. Int. Med. Trop. Sao Paulo. $15: 1-9$.

15.- Yarzabal, L., Albornoz, M., Cabral, N. (1978). Specific doble difussion microtecnique for the diagnosis of Aspergillosis and paracoccidioidomycosis using monospecific antisera. Sabouradia. 16: 55-62. 